\title{
Fractional step methods for the Navier-Stokes equations on non-staggered grids
}

\author{
S. W. Armfield* R. Street ${ }^{\dagger}$
}

(Received 7 August 2000)

\begin{abstract}
The Navier-Stokes equations are solved on a non-staggered grid using a semi-implicit fractional step method in both iterative and noniterative form. It is shown that the iterative scheme in standard form is second-order accurate in timei, but is very slow to integrate as a

* Department of Mechanical and Mechatronic Engineering, University of Sydney, NSW 2006, AustraliA. mailto:armfield@mech.eng.usyd.edu.au

$\dagger$ Environmental Fluid Mechanics Laboratory, Stanford University, California, USA. mailto:street@cive.stanford.edu

${ }^{0}$ See http: //anziamj . austms .org. au/V42/CTAC99/Armf for this article and ancillary services, (c) Austral. Mathematical Soc. 2000. Published 27 Nov 2000.
\end{abstract}


result of the non-elliptic pressure coupling at the grid scale. Inclusion of additional terms into the pressure correction equation for the iterative scheme ensures an elliptic pressure coupling at the grid scale, but introduces a first order in time error into the scheme, leading to a reduction in solution accuracy. The non-iterative scheme is shown to be second order accurate in time in standard form and to be considerably more efficient than the iterative scheme.

\section{Contents}

1 Introduction

C136

2 Method

C138

2.1 Iterative method . . . . . . . . . . . . . C140

2.2 Accuracy...................... C142

2.3 Pressure correction method . . . . . . . . . . . . C144

2.4 Discretisation ................ . . C145

3 Results

C146

4 Conclusions

C151

References

C153 


\section{Introduction}

Time integration of the Navier-Stokes equations is often carried out by means of the fractional-step procedure, first suggested by Harlow and Welch [1] and Chorin [2] . With Chorin's method at each time step an incomplete form of the momentum equations is integrated to yield an approximate velocity field, which will in general not be divergence free, then a correction is applied to that velocity field to produce a divergence free velocity field. The correction to the velocity field is an orthogonal projection in the sense that it projects the initial velocity field onto the divergence free field without changing the vorticity. This step is called the projection step, and schemes that use this approach are often called projection methods. The original Chorin method was modified for use with finite volumes defined on a staggered grid by Kim and Moin [3], and has since been used by many researchers for the simulation of unsteady flows [4]. The basic projection method drops the pressure gradients from the momentum equations and requires the use of special boundary conditions for the intermediate velocity field to ensure second order in time behaviour.

An alternative to the basic projection method is the pressure correction method, which is similar to the basic projection method, but with the pressure gradient term retained in the momentum equations. The Poisson's equation is then solved for a pressure correction which is used to correct the intermediate velocity field and enforce continuity. Both Van Kan [5] and Bell and Colella [6] have suggested and analysed pressure correction methods of 
this type. The schemes of Van Kan and Bell and Colella were non-iterative in the sense that the momentum and pressure correction equation are only solved once at each time step. Van Kan presented results for the NavierStokes equations showing that the pressure correction scheme was second order in time on a staggered grid, while Bell and Colella presented no time accuracy results. Gresho [7] carried out a detailed analysis of projection methods, labelling the basic method P1 and the pressure correction method P2. It was demonstrated analytically that P1 with physical boundary conditions for the intermediate velocity was first order in time whereas P2 was second order in time. The pressure correction approach can also be applied iteratively, whereby the momentum and pressure correction equation system is solved repeatedly at each time step, ensuring that any error associated with the single iteration is minimised.

Recently Armfield and Street examined a number of fractional step methods on staggered grids $([8,9])$. It was shown that the basic projection method with modified intermediate velocity boundary, the single step pressure correction method and the iterative pressure correction method all give second order in time behaviour. The iterative method was the most accurate but least efficient of the methods tested, while the projection method with modified boundary conditions and the single step pressure correction method with physical boundary conditions gave approximately equal accuracy and efficiency.

In this paper the iterative and single step pressure correction methods are applied on a non-staggered grid. On a non-staggered grid the coupling 
between the pressure gradient and divergence terms is non-elliptic at the grid scale, reducing the efficiency of the scheme and allowing growth in the grid scale pressure mode. A number of methods have been suggested for recovering grid-scale ellipticity for iterative schemes, all of which either explicitly or implicitly include a biharmonic pressure operator in the continuity equation, introducing an additional discretisation error [10,11]. These approaches have typically been used with implicit discretisations of the momentum equations and iterative solutions of the momentum/pressure equations. In this paper we will examine the behaviour of the semi-implicit discretisation in which the advection terms are approximated explicitly using Adams-Bashforth, and the viscous terms implicitly using Crank-Nicolson. Both non-elliptic and elliptic iterative schemes will be considered as well as the non-iterative pressure correction approach.

\section{Method}

The governing equations are the Navier-Stokes equations in unsteady incompressible non-dimensional form,

$$
\begin{gathered}
u_{t}+(u \cdot \nabla) u=-\nabla P+\frac{1}{R e} \nabla^{2} u, \\
\nabla \cdot u=0,
\end{gathered}
$$

where $u$ is the velocity, $P$ the pressure and $R e$ the Reynolds number. 
The continuous equations are discretised using Adams-Bashforth for the advective terms and Crank-Nicolson for the diffusive terms, giving the system,

$$
\begin{aligned}
\frac{v^{n+1}-v^{n}}{\Delta t}+\left[\frac{3}{2} H\left(v^{n}\right)-\frac{1}{2} H\left(v^{n-1}\right)\right] & =-G p^{n+1}+\frac{1}{2 R e} L\left(v^{n+1}+v^{n}\right), \\
D v^{n+1} & =0,
\end{aligned}
$$

where $(v, p)$ are the discrete velocity and pressure respectively, $H$ is the discrete advection operator, $G$ the discrete gradient, $L$ the discrete Laplace operator and $D$ the discrete divergence. This is a second order in time discretisation, using an explicit scheme for the advection terms and an implicit scheme for the diffusion terms. Fractional-step methods integrate equations (3) and (4) in a segregated manner, that is the momentum equations are first solved for the velocity, and some form of Poisson's equation is then solved for the pressure. The Poisson's equation is constructed from the momentum equation and the continuity equation and, as well as providing the pressure, also acts to enforce continuity.

The schemes considered in this paper first obtain an estimate for the velocity at the $n+1$ time level, by solving an approximation of equation (3), and then solve a Poisson's equation that is constructed by applying a correction to the estimated velocity field, requiring that it satisfy continuity. The iterative pressure correction method is the most general in the sense that it attempts to provide an exact solution to the discrete equations (3) and (4), at least to the degree of accuracy of the convergence criterion specified. The non-iterative pressure correction method is functionally identical to the iterative pressure correction method, but with the momentum and Poisson's 
equations being solved only once at each time step. In the rest of the paper for brevity the iterative pressure correction method will be denoted the iterative method, and the non-iterative pressure correction method will be denoted the pressure correction method.

\section{$2.1 \quad$ Iterative method}

Equation (3) is solved, using the best current value for $p^{n+1}$, to obtain $v^{*}$, an approximation to $v^{n+1}$, that is

$$
\frac{v^{*}-v^{n}}{\Delta t}+\left[\frac{3}{2} H\left(v^{n}\right)-\frac{1}{2} H\left(v^{n-1}\right)\right]=-G p^{n+1}+\frac{1}{2 R e} L\left(v^{*}+v^{n}\right) .
$$

This approximate velocity will not initially satisfy continuity. A correction is then applied of the form,

$$
v^{n+1}=v^{*}-\Delta t G P c,
$$

where $P c$ is a pressure correction, such that the resulting $v^{n+1}$ does satisfy continuity. An equation for $P c$ is constructed by substituting equation (6) into the continuity equation (4), to give,

$$
L P c=D v^{*} / \Delta t .
$$

The discrete divergence operator $D$ is obtained as,

$$
D^{i j}=\frac{\left(U^{i+1 / 2}-U^{i-1 / 2}\right)^{j}}{h}+\frac{\left(V^{j+1 / 2}-V^{j-1 / 2}\right)^{i}}{h}
$$


with $h$ the uniform grid spacing and $\left(U^{i+1 / 2}, V^{j+1 / 2}\right)$ the cell face or internodal velocities defined as,

$$
\begin{aligned}
& U^{i+1 / 2, j}=\frac{\left(U^{i+1}+U^{i}\right)^{j}}{2}+R^{i+1 / 2, j}, \\
& V^{i, j+1 / 2}=\frac{\left(V^{j+1}+V^{j}\right)^{i}}{2}+R^{i, j+1 / 2} .
\end{aligned}
$$

The $R$ terms represent a modification to the standard linear interpolation and are included to recover grid scale ellipticity [10]. They are defined as,

$$
\begin{aligned}
& R^{i+1 / 2, j}=\Delta t\left[\frac{p^{i+2}-p^{i}}{2 h}+\frac{p^{i+1}-p^{i-1}}{2 h}\right]^{j} / 2-\Delta t\left[\frac{p^{i+1}-p^{i}}{h}\right]^{j}, \\
& R^{i, j+1 / 2}=\Delta t\left[\frac{p^{j+2}-p^{j}}{2 h}+\frac{p^{j+1}-p^{j-1}}{2 h}\right]^{i} / 2-\Delta t\left[\frac{p^{j+1}-p^{j}}{h}\right]^{i} .
\end{aligned}
$$

The Laplace operator $L$ is discretised as a compact $h$ operator, that is

$$
L P c=\frac{\left(P c^{i+1}-2 P c^{i}+P c^{i-1}\right)^{j}}{h^{2}}+\frac{\left(P c^{j+1}-2 P c^{j}+P c^{j-1}\right)^{i}}{h^{2}} .
$$

Once $P c$ is obtained, the velocity is corrected and the pressure is updated using the pressure correction as,

$$
\begin{aligned}
U^{i j, n+1} & =U^{i j, *}-\Delta t \frac{\left(P c^{i+1}-P c^{i-1}\right)^{j}}{2 h}, \\
V^{i j, n+1} & =V^{i j, *}-\Delta t \frac{\left(P c^{j+1}-P c^{j-1}\right)^{i}}{2 h}, \\
& p^{n+1}=p^{n+1}+P c .
\end{aligned}
$$


Equation (5) is then solved again using the updated pressure to obtain a new estimate of the velocity at the $n+1$ time level, and that velocity again corrected to enforce modified continuity and provide a pressure correction. This process is repeated until the modified divergence of the velocity after the solution of equation (5) satisfies a predefined value. The solution is then said to be converged and the integration continues to the next time step. For the first iteration at each time step $p^{n+1}$ is set equal to $p^{n}$.

In practice this scheme is most efficient if it is not required that the pressure correction step results in a velocity field that satisfies the modified divergence free condition at each iteration, rather it is only required that the modified divergence error be reduced, and over a number of momentum/pressure correction iterations the velocity will approach the modified divergence free condition.

\subsection{Accuracy}

The $R$ terms will combine in the divergence operator to produce an error of the form,

$$
\begin{array}{r}
E=\frac{\Delta t}{4 h^{2}}\left(\left[p^{i+2}-4 p^{i+1}+6 p^{i}-4 p^{i-1}+p^{i-2}\right]^{j}+\right. \\
\left.\left[p^{j+2}-4 p^{j+1}+6 p^{j}-4 p^{j-1}+p^{j-2}\right]^{i}\right),
\end{array}
$$


which is a second order finite difference approximation for,

$$
\Delta t h^{2}\left(p_{x x x x}+p_{y y y y}\right) .
$$

The divergence has therefore been modified by the inclusion of the fourth derivative of the pressure multiplied by $\Delta t h^{2}$. At the completion of the time step the solution will satisfy equation (3), but will not satisfy equation (4) rather it will satisfy the modified form of equation (4) with the additional pressure term given above included. Although this will still be a consistent approximation to equation (4), the inclusion of this first order term will mean that the scheme will not be second order in time despite the use of a second order discretisation for the momentum equation and the iterative nature of the solution.

The use of non-staggered schemes of this type, where the inter-nodal or cell face velocity is obtained in the form given above, was first suggested by Rhie and Chow [12]. This method later came to be known as the momentum interpolation method and is now widely used. Armfield [10] showed that the success of the Rhie and Chow method, as well as many other similar schemes, was because the inclusion of additional terms ensured that grid scale ellipticity was retained. Analysis of all these schemes also showed that they had the effect, either explicitly or implicitly, of including the fourth derivative pressure terms into the divergence, as shown above. The resultant pressure is then forced to satisfy an implied Poisson equation with compact discretisation of the Laplace operator of the form given above in eqn. (8).

If the $R$ terms are not included the repeated solution of the coupled mo- 
mentum/pressure correction equations means that a solution is being sought in which the pressure will satisfy an implied Poisson equation with a sparse discretisation of the Laplace operator of the form,

$$
L p=\frac{\left(p^{i+2}-2 p^{i}+p^{i-2}\right)^{j}}{4 h^{2}}+\frac{\left(p^{j+2}-2 p^{j}+p^{j-2}\right)^{i}}{4 h^{2}} .
$$

This approach includes no additional error term into the continuity equation, but uncouples the grid scale pressure term, leading to poor performance. Results will be presented below for the standard iterative scheme, including the $R$ terms, and for the non-elliptic iterative scheme, without the $R$ terms.

\subsection{Pressure correction method}

The pressure correction method is similar to the iterative method, but with only a single iteration carried out at each time step. The discrete momentum equation is solved to obtain $v^{*}$ using the $n t h$ time-level pressure, as with the first iteration of the iterative method. A Poisson pressure correction equation is solved and the $v^{*}$ field and pressure corrected as with the iterative method, but without the $R$ terms included. The integration then continues to the next time step.

Using this approach the Poisson's pressure correction equation is not constructed directly from the discrete form of the continuity equation and the velocity corrections. The result is that the corrected nodal velocity field 
will not exactly satisfy continuity, substitution of $v^{n+1}$ into the continuity equation shows that there will be an error of the form

$$
E=\Delta t h^{2}\left(P c_{x x x x}+P c_{y y y y}\right)
$$

This is similar to the divergence error obtained with the iterative method when the $R$ terms are included, but is now dependent on the pressure correction. As $P c \sim \Delta t$ this error is expected to behave as $\Delta t^{2}$.

\subsection{Discretisation}

The above schemes are defined on a non-staggered grid using finite volumes, with standard second-order central differences used for the viscous terms, pressure gradient and divergence terms. The QUICK third-order upwind scheme is used for the advective terms [13]. Using QUICK the equations are discretised in conservative form, and it is necessary to specify the advective velocities at the inter-nodal cell face boundaries. A straightforward linear interpolation will produce a cell face velocity field with the divergence errors noted above incorporated in it, which may lead to a further degradation of the accuracy of the solution. To avoid this a divergence free cell face velocity field is used for the advecting velocity. The momentum equations are solved using an ADI scheme and the preconditioned GMRES method has been used to solve the Poisson pressure correction equation. 


\section{Results}

Results have been obtained for natural convection cavity flow which requires the inclusion of an additional temperature transport equation and a buoyancy term in the momentum equation for the vertical component of velocity. The temperature equation is solved using Adams-Bashforth for the advective terms and Crank-Nicolson for the diffusion terms in the same manner as that used for the momentum equations. Initially the fluid in the square cavity is stationary and isothermal at temperature $T=0$. At time $t=0$ the left and right walls are instantaneously heated and cooled to $\Delta T / 2$ and $-\Delta T / 2$ respectively, with the top and bottom boundaries adiabatic. All boundaries are no-slip. The control parameters for this flow are the Rayleigh number $R a$ and the Prandtl number $P r$. The Rayleigh number $R a=g \alpha \Delta T H^{3} / \nu \kappa$, with $g$ gravity, $\alpha$ the coefficient of thermal expansion, $H$ the height of the cavity, $\nu$ the kinematic viscosity and the thermal diffusivity $\kappa=\nu / \operatorname{Pr}$. The results presented were obtained with $R a=6 \times 10^{5}$ and $\operatorname{Pr}=7.5$.

A $50 \times 50$ uniform mesh was found to provide sufficient spatial resolution and has been used for all results presented here. The flow was integrated from $t=0$ to $t=2$ for time-steps in the range $\Delta t=0.003125$ to 0.1 , and the error expressed as the $L_{2}$ norm of the difference of the test solution and a solution obtained with a time step of $\Delta t=0.00078125$, also integrated from $t=0$ to $t=2$. Times have been non-dimensionalised using the boundary layer start-up time for the natural convection cavity. Total time to steady state for the cavity is an order of magnitude greater than the boundary layer 
start up time.

Further details of the natural convection flow may be found in Patterson and Armfield [14] and Armfield and Patterson [15]. Figure 1 contains the streamfunction and temperature contours at time $t=2$. The streamfunction shows the recirculation associated with each of the thermal boundary layers that form on the heated and cooled walls. The flow continues to develop from this stage with hot and cold intrusions ejected from the boundary layers travelling across the horizontal boundaries and stratifying the interior of the domain, and the two recirculation coalescing to form a single cavity scale recirculation.

For each of the methods and time steps results have been obtained with convergence criterion ranging from $1 \times 10^{-4}$ to $1 \times 10^{-10}$ in order-of-magnitude steps. The solution was considered converged at each time step when the integral over the domain of the absolute residual of the continuity equation was less than the convergence criterion. In this way it was possible to determine which was the appropriate convergence criterion for each method and time step to ensure as accurate as possible a solution was obtained. The results presented are those for which a further reduction of the criterion by an order of magnitude led to a less than one percent change in the solution accuracy. This degree of accuracy was obtained with different criterion for each method and each time step, ranging from $1 \times 10^{-6}$ for the iterative method with time step $\Delta t=0.1$ to $1 \times 10^{-9}$ for the pressure correction method at time step $\Delta t=0.003125$. For the iterative methods each integration of the Poisson's pressure correction equation was halted after five iterations of the 

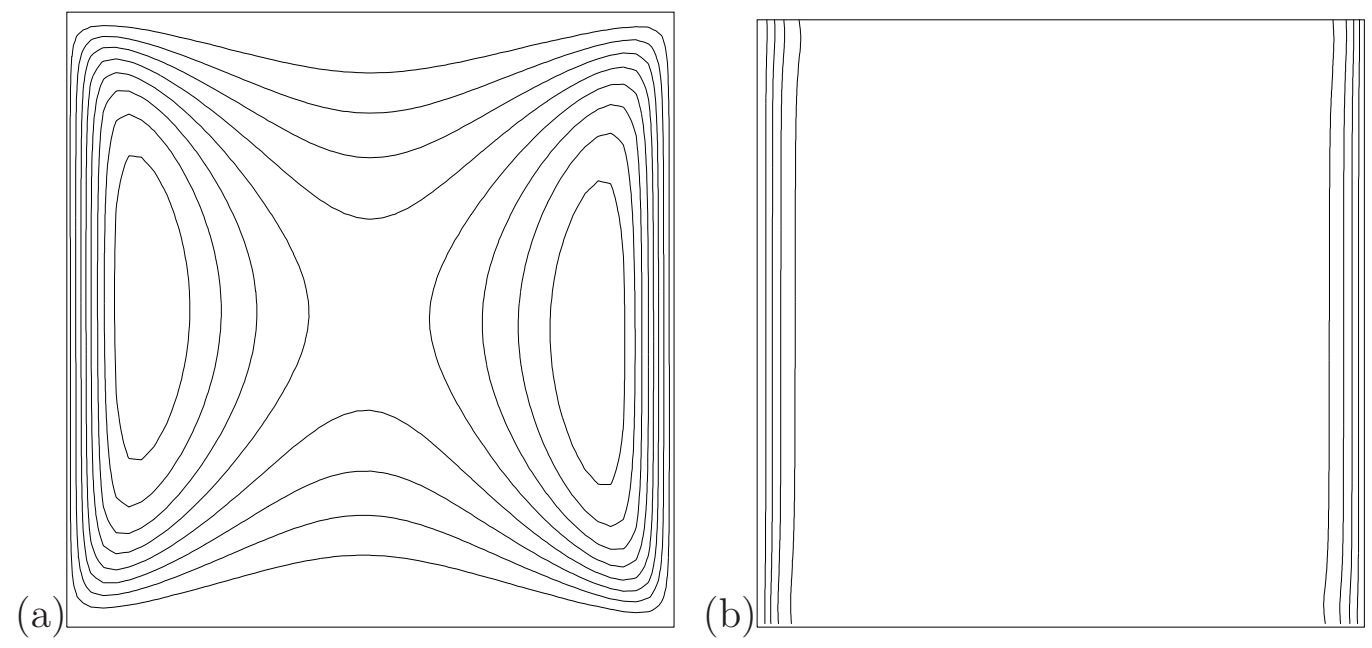

FiguRE 1: (a) Streamfunction contours, and (b) temperature contours. 

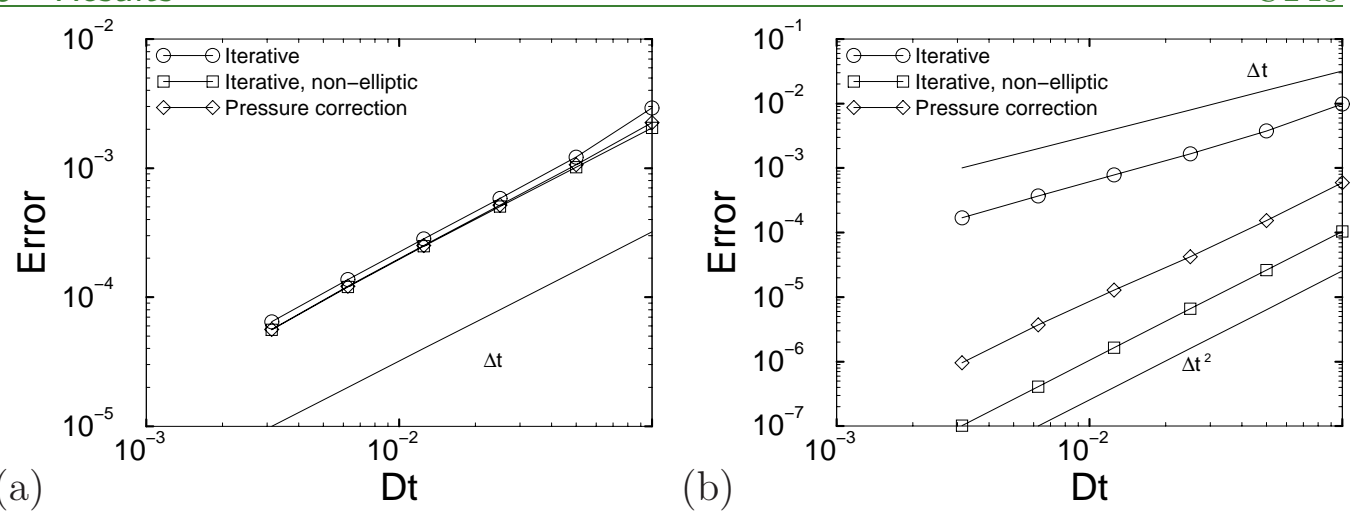

Figure 2: (a) Pressure error, and (b) U-velocity error.

GMRES procedure, regardless of the accuracy of the solution at that stage of integration. The divergence test is applied to the iterative method after the momentum equations have been solved, and it was required that at each time step two iterations of the momentum/pressure correction cycle were carried out, regardless of the divergence after the first solution of the momentum equations.

Figures 2 to 3 contain the error plotted against the time step for the pressure, U-velocity, $\mathrm{V}$-velocity and temperature respectively, for the pressure correction and iterative schemes. All schemes have order $\Delta t$ error for the pressure providing nearly identical results, as is expected for solvers of this type [16]. An order $\Delta t$ line is included in the figure for comparison. 

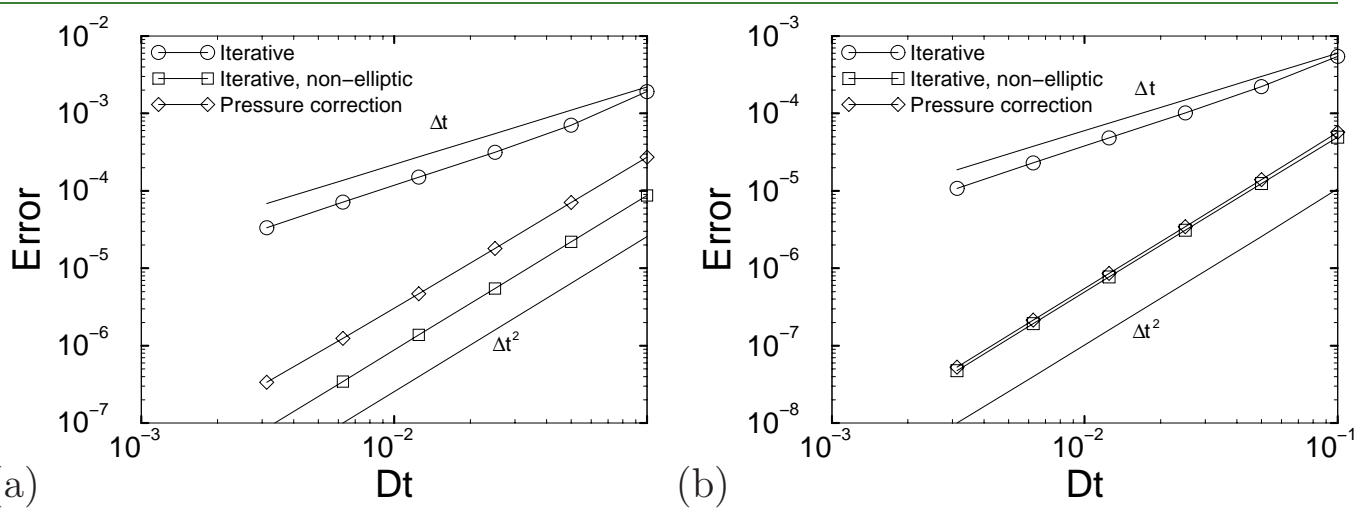

Figure 3: (a) V-velocity error, and (b) temperature error.

For the $\mathrm{U}$ and $\mathrm{V}$ velocities the standard iterative method gives a first order in time solution while the non-elliptic and pressure correction methods give second order in time behaviour. Both order $\Delta t$ and $\Delta t^{2}$ lines are included in the figures for comparison. The non-elliptic result is the most accurate, with an error about half that of the pressure correction error. Even at the largest time step the pressure correction method is an order-of-magnitude more accurate that the standard iterative method.

The temperature results show the same time behaviour and relative accuracy as the velocity results, with the exception that now the pressure correction and non-elliptic iterative solutions are almost identical. At the largest time step the pressure correction and non-elliptic iterative errors are an orderof-magnitude smaller than the error of the standard iterative method, with 
this difference considerably larger at the smaller time-steps. Again both order $\Delta t$ and $\Delta t^{2}$ lines are included in the figures for comparison.

Run times have been obtained for each of the methods, and are presented in Figure 4. The timings are processor times obtained running in double precision on a DEC 3000-700, and are shown as CPU time in seconds on the horizontal axis. The error for each method is the average of the velocity and temperature errors at each of the time steps for which results were obtained, and is shown on the vertical axis. Presenting the results in this form means that it is possible to compare the $\mathrm{CPU}$ time required for each method to achieve a given accuracy, and thus to assess the comparative efficiency of each method. The pressure correction method is clearly the most efficient of the three schemes, with the CPU times for the standard iterative method several times those of the pressure correction method, and the non-elliptic iterative method up to an order-of-magnitude greater.

\section{Conclusions}

The non-elliptic iterative scheme is the most accurate of those tested but is very inefficient. This was also observed for the fully implicit non-staggered iterative scheme detailed in Armfield [11]. The standard iterative scheme, with the $R$ terms included, requires considerably less CPU time but still performs relatively poorly because of the first order in time error included in the continuity equation, which considerably reduces the accuracy of the 


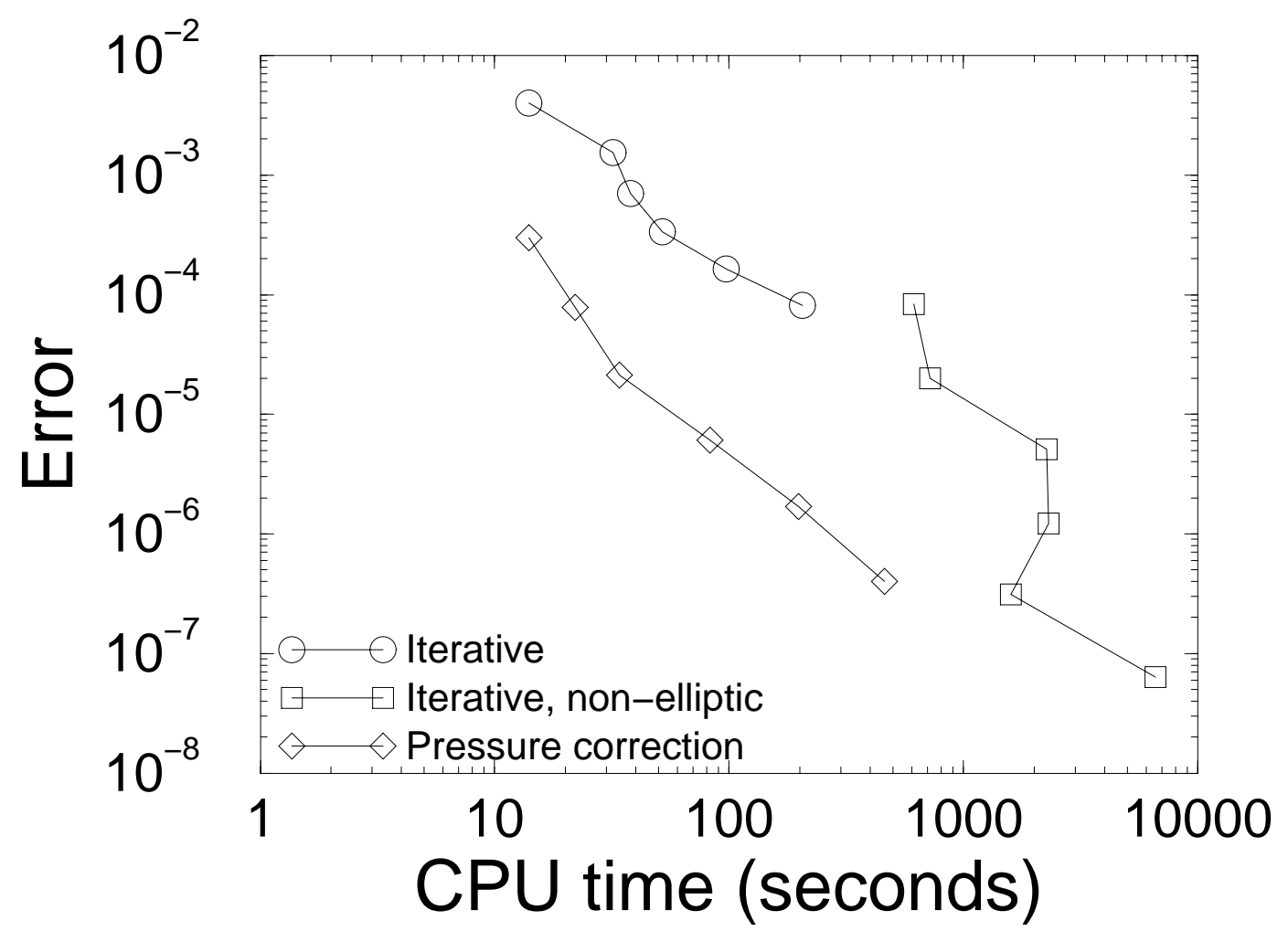

Figure 4: Timing results. 
scheme.

The pressure correction method produces second order in time accuracy and is the most efficient of the schemes tested by a considerable margin. The scheme does not attempt to satisfy an implied pressure correction equation at each time step, however the grid scale pressure is uncoupled as with the nonelliptic iterative scheme, which can result in grid scale pressure oscillations. No such oscillations were observed with the natural convection flow tested, even when the Rayleigh number was increased to $6 \times 10^{8}$ and integration was continued to steady state. If such oscillations were to occur they could be readily controlled by storing only the pressure gradient, which automatically filters the grid scale, for use in the momentum equations. It is apparent from the results presented here that the problem with the non-elliptic scheme is not the possible generation of a grid-scale pressure mode, but that the scheme is very inefficient.

Acknowledgment: the authors acknowledge the support of the UPS visiting professor scheme in Civil and Environmental Engineering at Stanford University and the Physical Meteorology Program of the National Science Foundation.

\section{References}


[1] F. Harlow and E. Welch. Numerical calculation of time-dependent viscous incompressible flow of fluid with free surface. Physics of Fluids, 8:2182-2189, 1965. C136

[2] A. J. Chorin. Numerical solution of the Navier-Stokes equations. Math. Comput., 22:745-762, 1968. C136

[3] J. Kim and P. Moin. Application of a fractional step method to incompressible Navier-Stokes equations. J. Comp. Physics, 59:308-323, 1985. C136

[4] Y. Zang, R. Street, and J. Koseff. A non-staggered grid, fractional step method for time-dependent incompressible Navier-Stokes equations in curvilinear coordinates. J. Comp. Physics, 114:18-33, 1994. C136

[5] J. Van Kan. A second order accurate pressure correction scheme for viscous imcompressible flow. SIAM J. Sci. Stat. Comput., 7:870-891, 1986. C136

[6] J. B. Bell and P. A. Colella. Second-order projection method for the incompressible Navier-Stokes equations. J. Comp. Physics, 85:257-283, 1989. C136

[7] P. Gresho. On the theory of semi-implicit projection methods for viscous incompressible flow and its implementation via a finite element method that also introduces a nearly consistent mass matrix. Part 1: theory. Int. J. Numerical Methods in Fluids, 11:587-620, 1990. C137 
[8] S. W. Armfield and R. Street. The fractional-step method for the Navier-Stokes equations on staggered grids: the accuracy of three variations. J. Comp. Physics, 153:660-665, 1999. C137

[9] S. W. Armfield and R. Street. An analysis and comparison of the time and accuracy of fractional-step methods for the Navier-Stokes equations on staggered grids. Int. J. Numerical Methods in Fluids, submitted, 1999. C137

[10] S. W. Armfield. Finite difference solutions of the Navier-Stokes equations on staggered and non-staggered grids. Computers and Fluids, 20:1-17, 1991. C138, C141, C143

[11] S. W. Armfield. Ellipticity, accuracy and convergence of the discrete Navier-Stokes equations. J. Comp. Physics, 114:176-184, 1994. C138, C151

[12] C. M. Rhie and W. L. Chow A numerical study of the turbulent flow past an airfoil with trailing edge separation. AIAA, paper 82-0988, 1982. C143

[13] B. P. Leonard. A stable and accurate convective modelling procedure based on quadratic upstream interpolation. Comp. Meth. Appl. Mech. and Eng., 19:59-98, 1979. C145

[14] J. C. Patterson and S. W. Armfield Transient features of natural convection in a cavity. J. Fluid Mech., 219:469-497, 1990. C147 
[15] S. W. Armfield and J. C. Patterson. Wave properties of natural convection boundary layers. J. Fluid Mech., 239:195-211, 1992. C147

[16] J. B. Perot. An analysis of the fractional step method. J. Comp. Physics, 108:249-253, 1993. C149 